\section{Tratamento de hérnia de disco lombar. Apresentação da técnica endoscópica e relato de dois casos clínicos.}

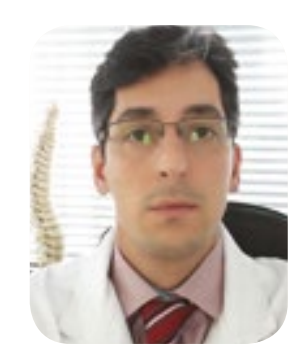

Tiago Argolo Bittencourt de Oliveira', Maurício Santos Gusmão', Rony Brito Fernandes', Maurício Guimarães Pimentel', Djalma Castro de Amorim Júnior

Dor lombar e ciática são os principais motivos ortopédicos de procura de assistência médica, sendo hérnia de disco lombar (HDL) a sua principal causa. Esses sintomas podem impactar de forma significativa as atividades diárias e a qualidade de vida do paciente. ${ }^{\top} \mathrm{A}$ $\mathrm{HDL}$ ocorre precocemente na cascata de degeneração da coluna, devido a uma falha do ânulo fibroso em conter o conteúdo gelatinoso do núcleo pulposo, que pode comprimir as raizes nervosas e provocar do lombar e radicular, associada ou não a déficit neuroló gico. Esses sintomas normalmente ocorrem de acordo com a distribuição dos dermátomos e miótomos da raiz neural comprimida, sendo as raízes de L4, L5 e S1 as mais acometidas. ${ }^{2,3} \mathrm{O}$ diagnóstico é firmado pela combinação do exame físico detalhado aos exames de imagem, como radiografia e ressonância magnética. Em geral, a HDL tem uma boa evolução com o tratamento conservador e cerca de 70 a $90 \%$ dos pacientes melhoram no período de 6 semanas a 3 meses. O tratamento medicamentoso envolve uso de anti-inflamatórios, analgésicos, corticoides e anticonvulsivantes, como pregabalina e gabapentina. A fisioterapia é fundamental e visa tanto analgesia como manobras de fortalecimento e estabilização do tronco. A infiltração foraminal com anestésico e corticoide também é uma ferramenta importante e pode reduzi significativamente a dor e aumentar o sucesso do tratamento conservador

Cerca de 10 a 30\% dos pacientes não respondem de forma satisfatoria às medidas conservadoras e, portanto, têm indicação de tratamento cirúrgico. Outras indicações para cirurgia incluem presença de síndrome da cauda equina ou de déficit neurológico significativo ou progressivo. A cirurgia tem objelivo de remover a hérnia e descomprimir as raízes nervosas. $\mathrm{A}$ técnica tradicional é a discectomia aberta, que consiste na remoção do fragmento herniado. Outra tecnica que vem ganhando cada vez mais espaço e aceitação é a discectomia endoscópica. Neste caso, utilizamos um endoscópio semelhante ao utilizado em artroscopias e laparoscopias, porém com uma diferença: o instrumental é introduzido por dentro do próprio endoscópio,

Estudos controlados e revisões sistemáticas têm demonstrado que a cirurgia endoscópica tem o mesmo resultado clínico da cirurgia tradicional, porém, com as vantagens da cirurgia minimamente invasiva. O endoscópio é introduzido por uma pequena incisão de menos de $1 \mathrm{~cm}$ e causa mínima dissecção muscular. Dess forma, o paciente tem menos dor pós-operatória, pode receber alta no mesmo dia, tem reabilitação mais rápida e pode retornar mais precocemente às suas atividades laborais. Além disso, complicações como infecção ou fístula liquórica são bem menos frequentes. ${ }^{7,8,9,10,1}$ Existem duas técnicas de discectomia endoscópica. A primeira descrita é a lateral transforaminal, mais utilizada para hérnias em L4-L5 ou níveis mais altos. A crista iliaca dificulta a realização desta técnica em L5-S1para hérnias centrais ou centro-laterais, que são as mais comuns. Com o intuito de tratar estas hérnias em L5-S1, foi desenvolvida a técnica posterior interlaminar. Esta também pode ser utilizada em níveis mais altos, porem com maior dificuldade, devido ao tamanho da janela interlaminar, que é mais ampla em L5-S e diminui progressivamente até L1-L2. Portanto, para níveis acima de L5-S1, a janela interlaminar normalmente tem que ser ampliada com uso de drill e outros instrumentos, que são introduzidos por dentro do pró-

\section{CASOS CLínICOS}

Os casos apresentados aqui foram tratados de (zabel a técnica endoscópica foi utilizada nos dois casos, visando os benefícios da cirurgia minimamente invasiva. prio endoscópio.?
Caso

Paciente do sexo masculino, 41 anos, dentista praticante de corrida de longas distâncias, foi internado devido lombociatalgia intensa há 3 dias, que 0 impedia de deambular. $\mathrm{Na}$ admissão, apresentava teste de Lasegue positivo, diminuição de sensibilidade no dorso do pe e diminuição de força (grau $4 \mathrm{em} \mathrm{5}$ ) para extensão do hálux esquerdo. As radiografias da coluna lombar revelavam diminuição do espaço discal L5-S1. A ressonancia magnetica evidenciou pequena protrusão central e volumosa hernia de disco foramina e extraforaminal em L5-S1 à esquerda, comprimindo e deslocando a raiz de L5 (figura 1). O paciente fez uso de medicações parenterais, teve acompanhamento com clínica da dor e com fisioterapia e foi realizada infiltração foraminal L5-S1 esquerda, sem melhora do quadro álgico, permanecendo acamado neste período. No $7^{\circ}$ dia de internamento, evoluiu com piora do défici de força do hálux esquerdo (grau $3 \mathrm{em} 5$ ).

Devido à piora do quadro com progressão do déficit neurológico, foi indicado tratamento cirúrgico para discectomia endoscópica transforaminal L5-S1. Essa técnica foi escolhida devido ao fato de a hérnia estar localizada no espaço foraminal e extraforamina.

O paciente foi posicionado em decúbito ventral com flexão dos quadris para diminuir a lordose lombar e, assim, aumentar o espaço foraminal. A anestesia re alizada foi a sedação consciente, para que o paciente pudesse dar feedback dos sintomas radiculares durante a cirurgia. Foi realizado discografia com contraste e azul de metileno para corar o fragmento herniado. Realizado então pequena incisão de cerca de $1 \mathrm{~cm}$ com anestesia local, $12 \mathrm{~cm}$ lateral à linha média, para introduçăo do fio guia, dilatador, cannula e endoscópio no espaço foraminal esquerdo de L5-S1 (figura 2). Fo utilizado intensificador de imagens para posicionamento apropriado do instumental. Então, sob visão endoscopica, foi identificado a raiz nervosa de $L 5$ esquerda comprimida pela hermia de disco, que foi removida com uma pinça introduzida por dentro do proprio endoscopio (figura 3). A incisão foi suturada com fio inabsorvivel e o fragmento da hernia de disco (medindo aproximadamente $1,7 \mathrm{~cm} \times 1,3 \mathrm{~cm}$ ) foi enviada para anatomia patológica (figura 4).

O paciente evoluiu com melhora completa da dor no pós-operatorio imediato, voltou a deambular no mesmo dia da cirurgia e teve alta hospitalar no dia seguinte. Apos 2 semanas, retomou parcialmente as atividades laborais e após 1 mês já estava trabalhando normalmente. Em 6 semanas retornou aos esportes, praticando natação e ciclismo. No pós-operatório apre- sentou disestesia no dermátomo de L5, melhorando completamente após 3 meses, período em que a força muscular também voltou ao normal.

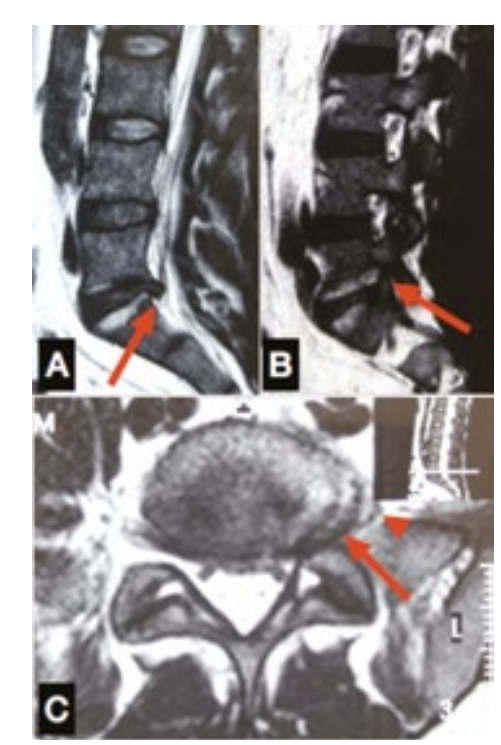

Figura 1 - Ressonância magnética da coluna lombar ponderada em T2. A: corte sagital central mostrando pequena protrusão central em L5-S1, sem compressão neural; B: corte sagital lateral revelando compressão foraminal L5-S1 esquerdo; C: corte axial evidenciando volumosa hérnia foraminal L5-S1 esquerda (seta), comprimindo e deslocando a raiz nervosa de L5 (cabeça da seta).

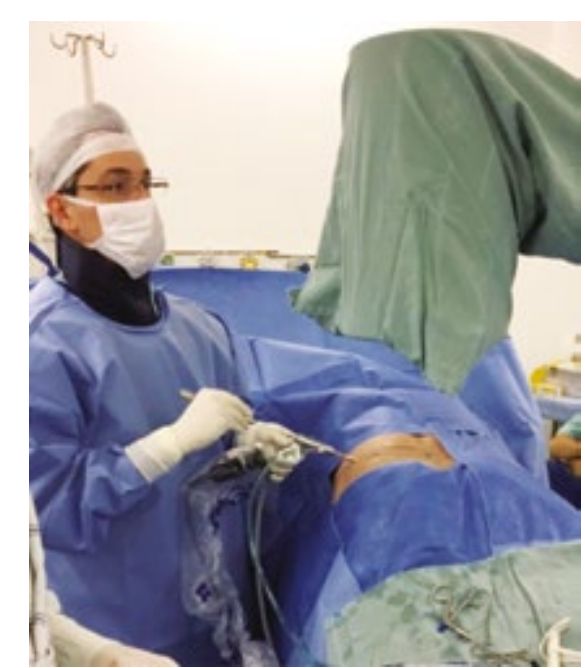

Figura 2 - Posicionamento do paciente e do endoscópio para a técnica transforaminal. 


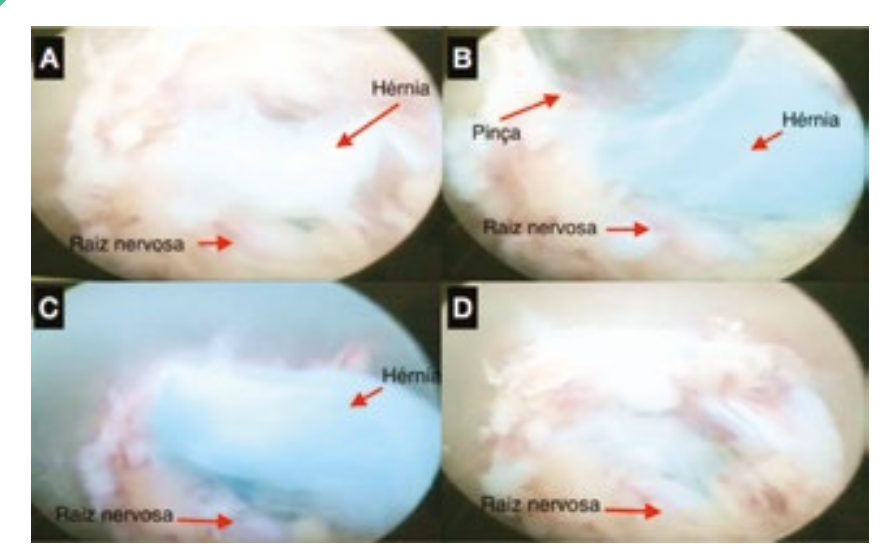

Figura 3 - Visão endoscópica pela técnica transforaminal. A: hérnia comprimindo a raiz nervosa; B: removendo a hérnia com uso de uma pinça introduzida por dentro do endoscópio; C: fragmento de hérnia de disco sendo removido; D: raiz de L5 livre.

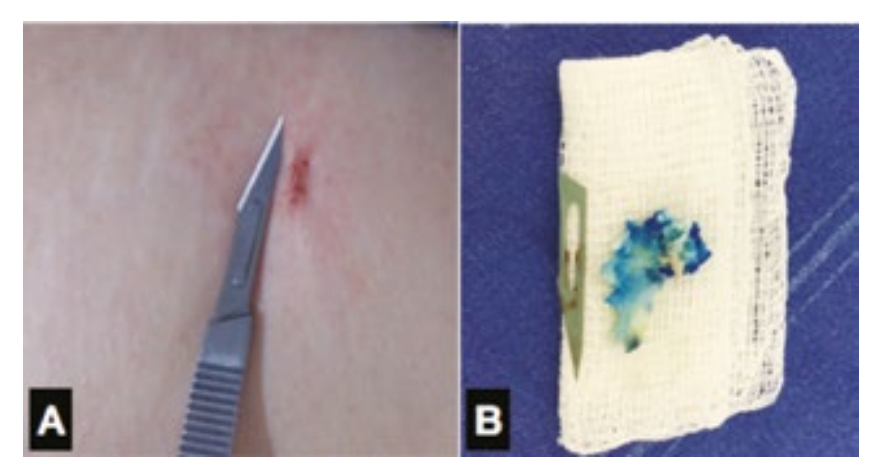

Figura 4 - Comparação com lâmina de bisturi n ${ }^{\circ} 11 . \mathrm{A}$ incisão suturada; B: fragmento de hérnia de disco removido.

Caso 2

Paciente do sexo masculino, 42 anos, empresário gosta de fazer trilha com quadriciclo. Se apresentou no consultório com queixa de dor lombar irradiando para o membro inferior esquerdo até o dorso do pé, há 2 meses, sem melhora com medicações e fisioterapia. Ao exame físico, apresentava teste de Lasegue positivo à esquerda. Sensibilidade e força sem alteraçōes. Radiografia mostrava redução do espaço discal L4-L5 e L5-S1. Ressonância magnética revelava hérnia centro-lateral esquerda em L4-L5, migrada caudal, comprimindo a raiz de $L 5$ no recesso lateral esquerdo (figura 5). Apresentava também protrusão central L5S1, porém sem compressão neural. Foi realizada in filtração foraminal L4-L5 esquerda (figura 6), apresen- tando melhora dos sintomas apenas por alguns dias, retornando na mesma intensidade.

Foi indicado tratamento cirúrgico após 2 meses e meio do início dos sintomas, devido à falha do tratamento conservador. A técnica escolhida foi a discectomia endoscópica interlaminar no nivel L4-L5, devido à hérnia estar migrada para caudal, o que dificultaria a técnica transforaminal.

o paciente fol posicionado em decúbito ventral com flexão dos quadris e anestesiado com sedação consciente. Foi realizada pequena incisão posterior na região lombar, de cerca de $1 \mathrm{~cm}$, com anestesia local, para introduçăo do dilatador, cânula e endoscópio no nível L4-L5 (figura 7). Aberto o ligamento amarelo com tesoura e observada raiz de L5 esquerda abaulada, devido a hernia de disco. Deslocamos a raiz nervosa medialmente com a própria cânula e visualizamos a hérnia de disco contida pelo ligamento longitudinal posterior, que foi cortado com a tesoura para que a hérnia pudesse ser removida com a pinça (figura 8). O paciente evoluiu com melhora completa da dor radicular e teve alta hospitalar no mesmo dia da cirurgia. Após 1 semana já estava trabalhando e após duas semanas estava andando de quadriciclo, embora tenha sido orientado a esperar 6 semanas para realizar tal atividade.

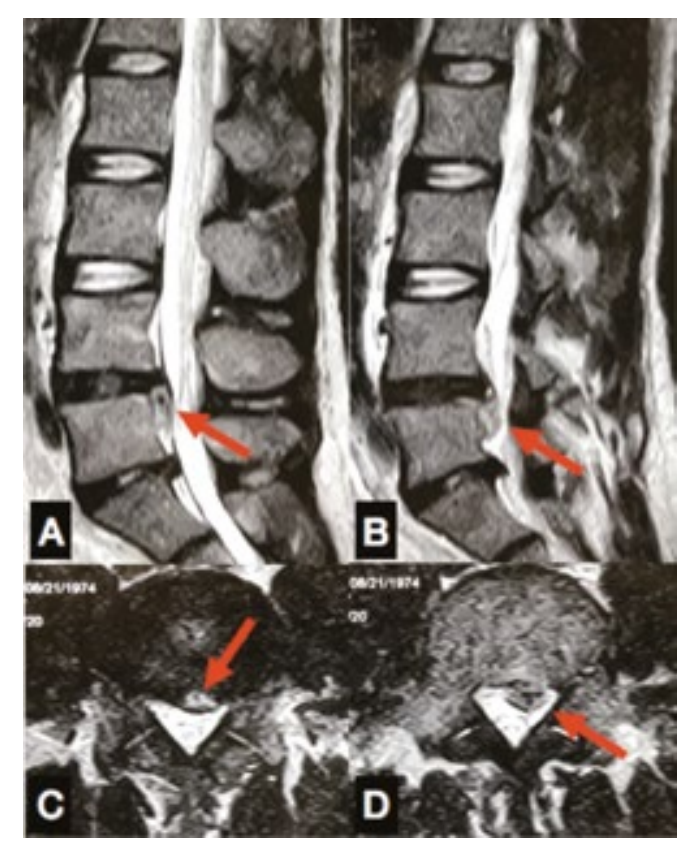

Figura 5 - Ressonância magnética da coluna lombar ponderada em T2. A e B: cortes sagitais sequenciais mostrando volumosa hérnia de disco L4-L5 migrada caudal (seta), além de pequena protrusão L5-S1; C: corte axial no nível L4-L5 revelando rotura do ânulo fibroso (seta), D: corte axial no nível L4-L5 revelando héria de disco centrolateral esquerda comprimindo a raiz nervosa de L5 (seta)

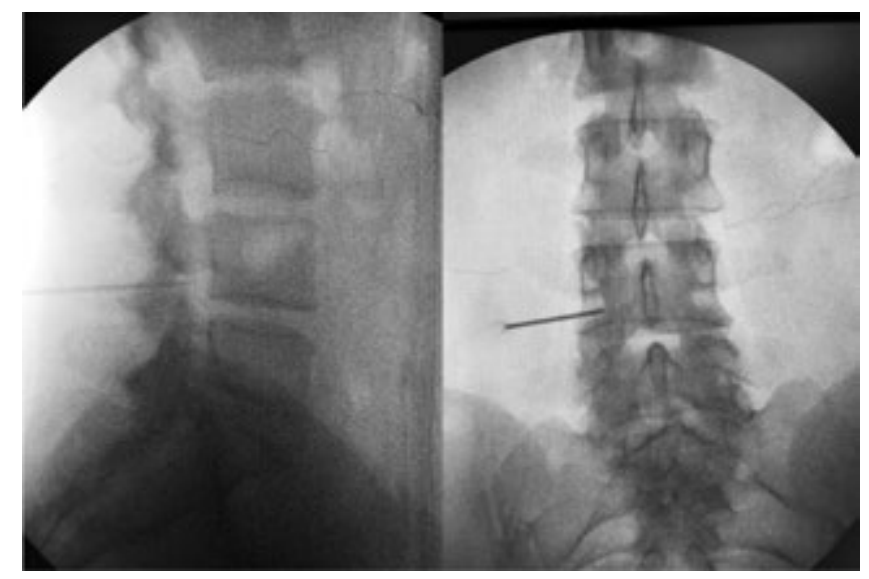

Figura 6 - Imagens da radioscopia em perfil e anteroposterior, com agulha posicionada em L4-L5 para infitração foraminal à esquerda.

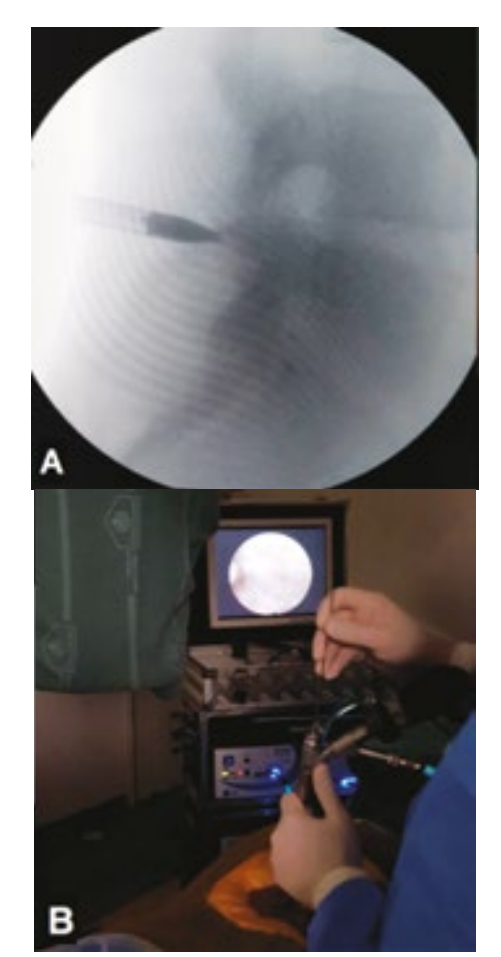

Figura 7 - A: radioscopia confirmando posicionamento do dilatador no nível L4-L5; B: posição posterior do endoscópio e a torre na frente do cirurgião.

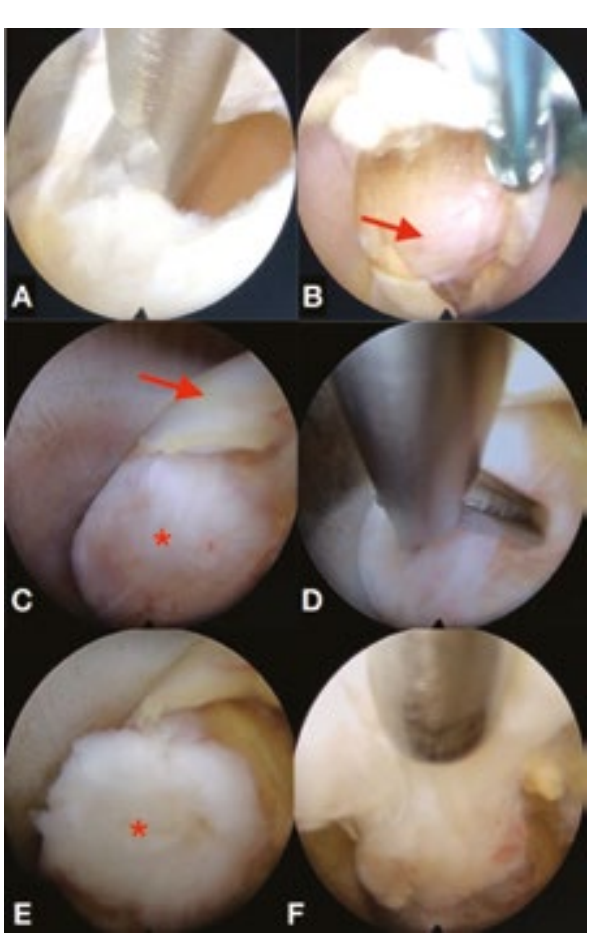

Figura 8 - visão endoscópica pela técnica interlaminar. A: utilizando tesoura para abrir o ligamento amarelo; $B$ : abaixo do ligamento amarelo vemos a raiz nervosa de L5 abaulada (seta); C: utilizando a cânula para deslocar a raiz medialmente (seta), observamos a hérnia de disco contida pelo ligamento longitudinal posterior (asterisco); D: cortamos o ligamento com a tesoura; $\mathrm{E}$ : hérnia de disco (asterisco) saindo pelo ligamento; $F$ : removendo a hernia com a pinça.

\section{PÓS-OPERATÓRIO}

O paciente normalmente recebe alta hospitalar no mesmo dia ou no dia seguinte da cirurgia, dependendo do horário em que esta foi realizada. As orientações de alta são: utilizar medicaçôes analgesicas, conforme necessário, evitar movimentos de liexăo do tronco, se abaixar ou pegar peso; utilizar colete de Putti baixo. $O$ colete tem por objetivo dar conforto ao paciente e ajudá-lo a evitar que realize as atividades descritas acima. Essas orientaçôes devem ser seguidas por 6 semanas. Após esse periodo, ele pode iniciar atividade fisica leve e sem impacto. Após 3 meses, não tem qualquer restrição. $O$ retorno às atividades laborais vai depender do tipo de trabalho que o paciente executa. Aqueles que exercem atividades de escritório podem voltar ao trabalho em 15 dias, enquanto que trabalhadores braçais devem aguardar por 2 ou até 3 meses. 


\section{REFERÊNCIAS}

1. Hart LG, Deyo RA, Cherkin DC. Physician office visits for low back pain. Frequency, clinical evaluation, and treatment patterns from a US national survey. Spine 1995;20:11-9.

2. Wilder DG, Pope MH, Frymoyer JW. The biomechanics of lumbar disc herniation and the effect of overload and instability. J Spinal Disord. 1988;1:16-32.

3. Smyth MJ, Wright VJ. Sciatica and the intervertebral disc: An experimental study. J Bone Joint Surg Am. 1958;40:1401.

4. Weinstein JN, Tosteson TD, Lurie JD, et al. Surgical vs nonoperative treatment for lumbar disk herniation: the Spine Patient Outcomes Research Trial (SPORT): a randomized trial. JAMA. 2006;296:24412450.

5. Vijay BV, Atul LB, Gregory EL, et al. Transforaminal Epidural Steroid Injections in Lumbosacral Radiculopathy: A Prospective Randomized Study. Spine. 2002;27:11-16.

6. Jacobs WC, Arts MP, van Tulder MW, et al. Surgical techniques for sciatica due to herniated disc, a systematic review. European spine journal : official publication of the European Spine Society, the European Spinal Deformity Society, and the European Section of the Cervical Spine Research Society 2012;21:2232-51.

7. Ruetten S, Komp M, Merk H, et al. Full-Endoscopic Interlaminar and Transforaminal Lumbar Discectomy Versus Conventional Microsurgical Technique: A Prospective, Randomized, Controlled Study. Spine. 2008;33:931-939.

8. Wu X, Zhuang S, Mao Z, et al. Microendoscopic Discectomy for Lumbar Disc Herniation: Surgical Technique and Outcome in 873 Consecutive Cases. Spine. 2006;31:2689-2694.

9. Birkenmaier C, Komp M,Ruetten S, et al. The current state of endoscopic disc surgery: review of controlled studies comparing full-endoscopic procedures for disc herniations to standard procedures. Pain Physician. 2013;16:335-44.

10. Gibson JNA, Cowie JG, Iprenburg M. Transforaminal endoscopic spinal surgery: The future 'gold standard' for discectomy? - A review. The Surgeon. 2012;10:290-296.

11. Nellensteijn J, Ostelo R, Bartels R, et al. Transforaminal endoscopic surgery for symptomatic lumbar disc herniations: a systematic review of the literature. Eur Spine J. 2010;19:181-204.
1- Serviço de Ortopedia do HSI.

Endereço para correspondência: tiagoargolo@gmail.com 